REFERENCE:

[1] Raschi E, Chighizola CB, Cesana L, et al. Arthritis Res Ther 2018;20:187

Disclosure of Interests: Cecilia Chighizola Speakers bureau: Inova Diagnostics, Daniela Privitera: None declared, Maria Orietta Borghi: None declared, Pier Luigi Meroni Consultant for: Inova, Thermofisher, Teofarma, Elena raschi: None declared

DOI: 10.1136/annrheumdis-2019-eular.5575

\section{AB0202 DYSPHAGIA: POOR PROGNOSIS IN IDIOPATHIC INFLAMMATORY MYOPATHIES}

Ana Carolina Costi, Claudia Pena, Adriana Testi, Pierina Sansinanea, Mercedes García. Hospital Interzonal General de Agudos Gneral José de San Martín, Buenos Aires, La Plata, Argentina

Background: Dysphagia has been reported to occur in $10 \%$ to $73 \%$ of these patients and can be present at any time during the disease process (1).

Objectives: The primary objective of the study was to evaluate the prevalence of dysphagia in a cohort of patients with idiopathic inflammatory myopathy (MII) and to evaluate factors associated with the presence of dysphagia. The secondary objective was to evaluate the factors associated with severe dysphagia.

Methods: Retrospective, observational study, which included patients with a diagnosis of MIl according to modified classification criteria of Bohan and Peter (1992-2018). Demographic data, clinical characteristics, laboratory data, autoantibodies, imaging studies, videodeglution, muscle biopsy and EMG were recorded.

Severe dysphagia was considered: one in which oral feeding was contraindicated and/or which required nasogastric tube feeding (SNG) either by clinical evaluation or by videodeglution study. The rest of the patients with dysphagia who did not present a contraindication to oral intake during the course of the disease were considered mild/moderate dysphagia. Results: $94 / 110$ patients were included, $76 \%$ female, mean age at diagnosis: 48 years $(S D \pm 14)$. Idiopathic dermatomyositis was the most frequent subtype of myopathy (64\%). Dysphagia occurred in 53/94 patients $(56.4 \%)$ and it was presented at the beginning of the disease in $31 / 94$ $(32 \%)$. Severe dysphagia was found in (22/94) $23 \%$.

When analyzing the clinical features of patients with myopathy and dysphagia, it was found that Idiopathic dermatomyositis was the most frequent $\mathrm{MII}$ in these patients $(71 \%)$. Patients with dysphagia presented: proximal muscle weakness $90 \%$, neck muscles weakness $47 \%$, and respiratory muscle weakness $27 \%$.

Treatment received: 90/94 (97\%) oral glucocorticoids, mean dose $48 \mathrm{mg}$ of prednisone (Range $4-100 \mathrm{mg}$.), pulses of Intravenous methylprednisolone was indicated in 25 patients $(27.5 \%)$. The main steroid sparing agents used were: $72 \%$ methotrexate, followed by $33 \%$ azathioprine.

Significant association was found between dysphagia and weakness of neck muscles, respiratory muscles, of glucocorticoid pulses, gamma globulin and mortality (data not shown). In the Logistic Regression analysis, no variable was independently associated with the presence of dysphagia.

When analyzing the relationship of severe dysphagia and factors associated, a significant association was found with the requirement of mechanical ventilation, hospitalization in an intensive care unit, serious infections, neoplasia and mortality (Table 1). In the multivariate analysis: no associated factors were found independently.

Table 1

\begin{tabular}{|c|c|c|c|c|c|}
\hline Variable & $\begin{array}{l}\text { severe dysphagia } \\
\text { (present) } n=22(\%)\end{array}$ & $\begin{array}{l}\text { severe dysphagia } \\
\text { (absent) } n=72(\%)\end{array}$ & $p$ & OR & IC \\
\hline $\begin{array}{l}\text { Weak neck } \\
\text { muscles }\end{array}$ & 15 (68\%) & $18(25 \%)$ & $<0.001$ & 8.86 & $\begin{array}{l}2.75- \\
8.86\end{array}$ \\
\hline $\begin{array}{l}\text { Weakness of } \\
\text { respiratory } \\
\text { muscles }\end{array}$ & 10 (45\%) & $6(8 \%)$ & $<0.001$ & 9.5 & 2.8-32 \\
\hline $\begin{array}{l}\text { pulses of } \\
\text { corticosteroids }\end{array}$ & 17 (77\%) & $8(11 \%)$ & $<0.001$ & 25 & $\begin{array}{l}7.5- \\
89.5\end{array}$ \\
\hline $\begin{array}{l}\text { intravenous } \\
\text { gammaglobulin }\end{array}$ & $10(45 \%)$ & 7 (9\%) & $<0.001$ & 7.73 & $\begin{array}{c}2.46- \\
24\end{array}$ \\
\hline $\begin{array}{l}\text { intensive care } \\
\text { unit }\end{array}$ & $8(36 \%)$ & $8(11 \%)$ & $<0.001$ & 4.57 & $\begin{array}{l}1.46- \\
14.26\end{array}$ \\
\hline $\begin{array}{l}\text { mechanical } \\
\text { ventilation }\end{array}$ & $6(27 \%)$ & $5(7 \%)$ & $<0.001$ & 5.02 & $\begin{array}{l}1.36- \\
18.55\end{array}$ \\
\hline death & $16(72 \%)$ & 10 (14\%) & $<0.001$ & 16.53 & 5.89- \\
\hline
\end{tabular}

\begin{tabular}{lccccc} 
neoplasms & $6(27 \%)$ & $9(12 \%)$ & $<0.001$ & 2.62 & $0.65-$ \\
serious infections & $11(50 \%)$ & $10(14 \%)$ & $<0,002$ & 4.95 & $1,71-$ \\
& & & & & 14 \\
\hline
\end{tabular}

Conclusion: in patients with MII 56\% course with dysphagia at some point in the evolution. Of which $23 \%$ of cases were characterized as severe dysphagia. Both dysphagia in general and severe dysphagia were associated with parameters of severity, high cost and poor prognosis However, in the analysis of multiple variables, this relationship could not be demonstrated.

\section{REFERENCE:}

[1] Mayo Clin Proc. • April 2007;82(4):441-447

Disclosure of Interests: ANA CAROLINA COSTI: None declared, Claudia Pena: None declared, Adriana Testi: None declared, Pierina Sansinanea: None declared, Mercedes García Grant/research support from: GSK, Speakers bureau: GSK

DOI: 10.1136/annrheumdis-2019-eular.6093

\section{AB0203 INTRAPLATELET ANGIOGENIC AND GROWTH FACTORS IN SYSTEMIC SCLEROSIS}

Bryan-Josué Flores Robles ${ }^{1}$, María-Alejandra Sánchez ${ }^{2}$, José Luís Bueno Cabrera $^{2}$, Lucía Nuñez Martín-Buitrago ${ }^{2}$, Cristina Gómez Casado ${ }^{3}$, Juan Mulero Mendoza ${ }^{2}$, Hilda Godoy ${ }^{2}$, Valvanera Pinillos ${ }^{1}$, Eztizen Labrador-Sánchez ${ }^{1}$, Juan Antonio López-Martín ${ }^{1}$, Angel Elena-lbáñez ${ }^{1}$, Leticia Merino-Meléndez ${ }^{1}$, José Luis Andréu Sánchez ${ }^{2} .{ }^{1}$ Hospital San Pedro, Logroño, La Rioja, Spain; ${ }^{2}$ Puerta de Hierro Hospital, Madrid, Spain; ${ }^{3}$ Institute of Applied Molecular Medicine (IMMA) San Pablo-CEU University Madrid, Molecular Medicine, Madrid, Spain

Background: Systemic sclerosis (SE) is a serious disease of the connective tissue characterized by dysfunction of the microcirculation. Angiogenesis is a complex process regulated by both angiogenic and angiostatic factors. Normally the functions of these factors are under an adequate balance, however, under certain conditions these factors can be induced initiating disorganized angiogenic phenomena.

Objectives: For the first time, the levels of intraplatelet growth and angiogenic factors (normalized by platelet number and volumen) are described in patients with systemic sclerosis.

Methods: We included 23 patients with systemic sclerosis (ACR/EULAR) and 16 controls. Patients underwent a platelet-apheresis to obtain platelet-rich plasma (PRP) and platelet-poor plasma (PPP). The samples were subjected to a freeze-thaw process to break the cell membranes and release the platelet content. Subsequently the various factors were measured (VEGF- $\alpha$, PDGF $\beta \beta$, HGF, FGF2, G-CSF, MCP-1, IL-1 $\alpha$, IL-1 $1 \beta$, IL- 8 , IL-6, IL-13). Finally, the results were normalized according to the value of the plaquetocrit (number and volumen of platelets). We compared the levels in platelet-rich plasma and in platelet-poor plasma. A cut-off value $p$ $<0.05$.

Results: The mean age of the patients was 52 years \pm 9.71 , with a duration of the disease of 8 years, $74 \%$ were patients with limited SE, $70 \%$ of the patients had pulmonary involvement. Both patientes and controls, the TFG- $\beta$ was 12 times more concentrated in PRP than in PPP $(p<0.0001)$, IL-1b 13 times more $(p<0.0001)$, IL-6 10 times more $(p$ $<0.0001)$, G-CSF 11 times more $(p<0.0001)$, VEGF- $\alpha$ more concentrated (11.6 times) $p<0.005$. When the PRP of the patients with scleroderma was compared with the PRP of the controls, only differences were found in the VEGF concentrations, being decreased in the patients with scleroderma respect to controls $(p<0.0001)$. Table 1 and 2 .

Conclusion: The levels of VEGF- $\alpha$ (intraplatelet) are lower in patients with SE vrs controls $(p<0.0001)$. The intraplatelet values of the growth and angiogenic factors are higher than the plasma (patients and controls), finally, the plasma levels of these factors are similar in patients compare with controls. In almost all studies until today the measured levels have been carried out in serum, which are not precise since, in serum, several cells and lysate residues can alter the values, therefore, quantification in plasma is important. The present work is the result of the doctoral thesis of the main author.

Table 1. Growth factors values (23 patients with SE and 16 controls)

\begin{tabular}{|c|c|c|c|}
\hline Factor & $\begin{array}{c}P P P(p g / \\
m L)\end{array}$ & $\begin{array}{c}P R P(p g / \\
m L)\end{array}$ & $p$ \\
\hline TFG- $\beta 1$ & 21149,7 & 237185,2 & $<0.0001$ \\
\hline IL-1 $1 \beta$ & 7,7 & 117 & $<0.0001$ \\
\hline
\end{tabular}




\begin{tabular}{lccc} 
IL-6 & 8,1 & 39,7 & $<0.0001$ \\
IL-8 & 2,4 & 3,4 & 0.169 \\
IL-13 & 5,4 & 7,82 & 0.005 \\
G-CSF & 20,9 & 278 & $<0.0001$ \\
HGF & 73,0 & 109,7 & $<0.001$ \\
MCP-1 & 64,8 & 0.01 \\
IL-1 $\alpha$ & 1,2 & 9,1 & $<0.001$ \\
FGF-2 & 2.9 & 25,5 & $<0.001$ \\
VEGF & 32,3 & 406,6 & $<0.001$ \\
PDGF- & 8,2 & 329,7 & $<0.001$ \\
$\beta$ & & & \\
\hline
\end{tabular}

Table 2. Values of growth factors in platelet rich plasma (PRP), $n$ patients $=23$, controls $=16$

\begin{tabular}{|c|c|c|c|}
\hline Factor & & $\begin{array}{c}\text { Mean }(p g / \\
m L)^{*}\end{array}$ & $p$ \\
\hline$\overline{T F G-\beta 1}$ & Patients & 220271,8 & 0,327 \\
\hline Controls & 261498,1 & & \\
\hline \multirow[t]{2}{*}{$I L-1 \beta$} & Patients & 111,3 & 0,492 \\
\hline & Controls & 125,3 & \\
\hline \multirow[t]{2}{*}{ IL-6 } & Patients & 41,4 & 0,575 \\
\hline & Controls & 37,2 & \\
\hline \multirow[t]{2}{*}{ IL-8 } & Patients & 2,4 & 0,159 \\
\hline & Controls & 2,4 & \\
\hline \multirow[t]{2}{*}{$I L-13$} & Patients & 8,1 & 0,574 \\
\hline & Controls & 7,4 & \\
\hline \multirow[t]{2}{*}{ G-CSF } & Patients & 282,0 & 0,820 \\
\hline & Controls & 272,2 & \\
\hline \multirow[t]{2}{*}{$H G F$} & Patients & 105,5 & 0,598 \\
\hline & Controls & 115,9 & \\
\hline \multirow[t]{2}{*}{$M C P-1$} & Patients & 66,9 & 0,841 \\
\hline & Controls & 61,8 & \\
\hline \multirow[t]{2}{*}{$I L-1 \alpha$} & Patients & 8,4 & 0,517 \\
\hline & Controls & 10,1 & \\
\hline \multirow[t]{2}{*}{ FGF-2 } & Patients & 29,4 & 0,257 \\
\hline & Controls & 19,9 & \\
\hline \multirow[t]{2}{*}{ VEGF } & Patients & 248,9 & $<0.0001^{* * *}$ \\
\hline & Controls & 633,3 & \\
\hline \multirow[t]{2}{*}{$P D G F-\beta$} & Patients & 270,6 & 0,12 \\
\hline & Controls & 414,7 & \\
\hline
\end{tabular}

\section{REFERENCE:}

[1] Choi JJ, et al. Elevated vascular endothelial growth factor in systemic sclerosis. J Rheumatol2003; 30: 1529-33.

Acknowledgement: Patients and bank blood secretary. Disclosure of Interests:

Bryan-Josué Flores Robles Grant/research support from: Transport and hotel, María-Alejandra Sánchez : None declared, José Luís Bueno cabrera : None declared, Lucía Nuñez Martín-Buitrago : None declared, Cristina Gómez Casado: None declared, Juan Mulero Mendoza: None declared, Hilda Godoy: None declared, Valvanera Pinillos: None declared, Eztizen Labrador-Sánchez: None declared, Juan Antonio López-Martín: None declared, Angel Elena-lbáñez: None declared, Leticia Merino-Meléndez: None declared, José Luis Andréu Sánchez: None declared DOI: 10.1136/annrheumdis-2019-eular.4619

\section{AB0204 ANTIBODY POSITIVE DERMATOMYOSITIS}

Yoshikazu Fujikawa, Kengo Akashi, Motoko Katayama, Mai Yamashita, Yoko Nose, Takaichi Okano, Yo Ueda, Sho Sendo, Akira Onishi, Jun Saegusa, Akio Morinobu. Kobe univesity graduate school of medicine, Rheumatology and Clinical Immunology, Kobe, Japan

Background: Anti-TIF1gamma antibody positive dermatomyositis(TIF1 $\gamma$-DM) has greater risk of complication with cancer.

Objectives: We aimed to analyze the clinical features of TIF1 $\gamma$-DM and find the difference between cancer-associated TIF1 $\gamma$-DM (CA-TIF1 $\gamma$-DM) and non-cancer-associated TIF1 $\gamma$-DM (nCA-TIF1 $\gamma$-DM).

Methods: We investigated the clinical data of idiopathic inflammatory myositis (IIM) patients received remission induction therapy in out hospital between January 2006 and December 2018.

Results: We treated 148 patients with IIM in this period, 9 cases of them were TIF $1 \gamma$-DM. Patients with CA-TIF $1 \gamma$-DM and nCA-TIF1 $\gamma$-DM were 4 and 5 cases, respectively. Average age at onset of 9 patients with TIF $1 \gamma$-DM was 63 years old. CA-TIF $1 \gamma$-DM was significantly older than nCA-TIF1 $\gamma$-DM (average:78.0 vs 57.4). All of 9 TIF1 $\gamma$-DM had distinctive skin rash and muscle weakness. Seven cases of them suffered from dysphagia. Interstitial lung disease was not seen in all patients with
TIF1 $\gamma$-DM. Serum creatine kinase (CK) levels of CA-TIF1 $\gamma$-DM was significantly higher than those of nCA-TIF1 $\gamma$-DM (average: $3405.5 \mathrm{IU} / \mathrm{L}$ vs 719.8IU/L) (Fig 1.). Among the TIF1 $\gamma$-DM patients, we administered glucocorticoid (GCs) for 8 cases, immunosuppresants for 4 cases, and intravenous immunoglobulins (IVIG) for 7 cases. All 4 cases of CA-TIF1 $\gamma$-DM were treated with GC and IVIG. All TIF $1 \gamma$-DM patients, especially 4 CATIF1 $\gamma$-DM patients, seemed to have good response to treatment in serum CK levels. Although serum CK levels of these 4 cases were normalized within a month (Fig1.), their muscle weakness, skin rash and dysphagia which was associated with quality of life and the prognosis were poorly improved.

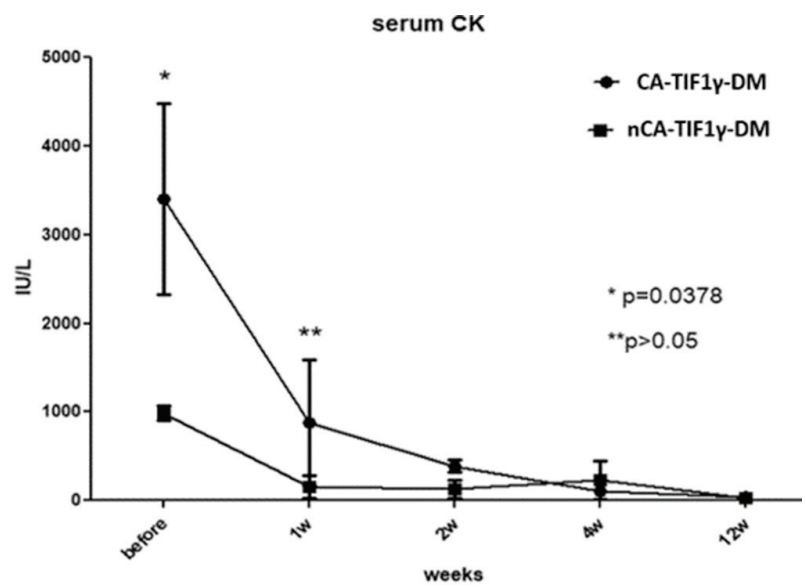

Figure 1: serum CK levels for 3months after treatment; Compared with cancer-associated TIF1Y-DM (CA-TIF1Y-DM) and non-cancer-associated TIF1 $Y$-DM (nCA-TIF1Y-DM)

Figure 1

Conclusion: In our investigation, the age of onset and serum CK levels were significantly different between CA-TIF $1 \gamma$-DM and nCA-TIF $1 \gamma$-DM. We report our investigation of the phenotype and treatment of anti TIF1gamma antibody positive dermatomyositis. TIF1 $\gamma$-DM with high serum CK levels, we should perform appropriate tests to check for cancer throughout our treatment, and the improvement of serum $\mathrm{CK}$ levels is not equal to the success of our treatment.

Disclosure of Interests: None declared

DOI: 10.1136/annrheumdis-2019-eular.6419

\section{AB0205 TREATMENT OF INFLAMMATORY MYOSITIS IN CLINICAL PRACTICE: ANALYSIS OF THE REGISTRY OF INFLAMMATORY MYOSITIS FROM THE MADRID COMMUNITY (REMICAM)}

Beatriz Joven-lbáñez ${ }^{1}$, Laura Nuño ${ }^{2}$, Francisco J. López-Longo ${ }^{3}$, Julia MartinezBarrio $^{3}$, Carmen Larena ${ }^{4}$, Valentina Maldonado ${ }^{4}$, Carmen Barbadillo ${ }^{5}$, Paloma García de la Peña ${ }^{6}$, Irene Llorente ${ }^{7}$, Eva Tomero Muriel ${ }^{7}$, Ana Pérez Gómez ${ }^{8}$, Henry Moruno ${ }^{8}$, Tatiana Cobo-lbáñez ${ }^{9}$, Raquel Almodovar ${ }^{10}$, Leticia Lojo ${ }^{11}$, María Jesús García de Yébenes ${ }^{12}$, Patricia Carreira ${ }^{1} .{ }^{1}$ University Hospital 12 de Octubre, Rheumatology, Madrid, Spain; ${ }^{2}$ Hospital La Paz-madrid, Madrid, Spain; ${ }^{3}$ Gregorio Marañón Hospital, Madrid, Spain; ${ }^{4}$ Hospital Ramón YCajal, Madrid, Spain; ${ }^{5}$ Hospital Puerta de Hierro-Majadahonda, Majadahonda, Spain; ${ }^{6}$ HM University Sanchinarro Hospital, Madrid, Spain; ${ }^{7}$ Hospital de La Princesa, Madrid, Spain; ${ }^{8}$ Hospital Príncipe de Asturias, Alcalá de Henares, Spain; ${ }^{9}$ Infanta Sofia University Hospital, San Sebastián de los Reyes, Spain; ${ }^{10}$ Fundación Hospital Alcorcón, Alcorcón, Spain; ${ }^{11}$ Hospital Universitario Infanta Leonor, Madrid, Spain; ${ }^{12}$ Instituto de salud musculoesquelética, Madrid, Spain

Background: Although glucocorticoids (GC) remain the cornerstone in the treatment of inflammatory myositis (IM), the management of these processes is not yet standardized. Experts recommend early use of immunosuppressants (IS), in order to prevent GC side effects. It is not known what is the actual management in clinical practice.

Objectives: To describe the management of IM in REMICAM registry, and to identify differences according to disease subtype (polymyositis (PM), or dermatomyositis (DM)), and to the year of diagnosis (before or after 2000).

Methods: All patients from REMICAM were included (1). A descriptive analysis of the different therapies (GC, IS, biologics, IV immunoglobulins 